\title{
Analogue model study of EM induction in elongated conductors-2D and 3D induction arrow responses
}

\author{
H. W. Dosso and J. Chen \\ Department of Physics and Astronomy, University of Victoria, Victoria B. C., Canada \\ (Received October 15, 1999; Revised February 2, 2000; Accepted March 21, 2000)
}

\begin{abstract}
Magnetic induction arrow responses of elongated conductors embedded in a uniformly resistive host earth and underlain by a conductive substratum are studied with the aid of laboratory analogue model measurements. The dependence on period, the overburden depth, and the underlying conductive basement depth, are examined for three different conductor lengths that were chosen so as to include the transition in the responses from two dimensional (2D) to those of three dimensional (3D) structures. The characteristic period $T c$ (the period at which the in-phase anomalous response of the elongated conductor model is maximum and the quadrature response is minimum) is found to depend on the conductor length, the overburden depth, and the conductive basement depth. A decrease in conductor length or conductive basement depth, each have the effect of decreasing both the response amplitude and the characteristic period, while increasing the overburden depth has the effect of increasing the characteristic period and decreasing the response amplitude. At short periods, say below the characteristic period, the responses appear to be roughly $2 \mathrm{D}$, and to become progressively more 3D-like with increasing period above the characteristic period.
\end{abstract}

\section{Introduction}

Electromagnetic induction in an anomalous conductor embedded in a resistive host earth continues to be of interest in regional geomagnetic surveys. One of the goals in geomagnetic surveys is to interpret the field measurements in order to define any anomalous structures present. The procedure would be to carry out an interpretation of the measurements by either, or both, inverting the data and forward numerical modelling. Although many well established two dimensional (2D) numerical methods are being used in forward modelling and inversion, this is less the case for 3D. The validity of various 2D methods for simplified models has been established in recent years by comparing the results with analytical calculations or with analogue model measurements. To date, it appears there are few if any 3D analytical solutions available to test the validity of 3D numerical modelling methods for cases of high conductivity contrasts. However, just as analogue model measurements have at times provided test results for $2 \mathrm{D}$ numerical methods, they can now also provide test values for examining the validity of $3 \mathrm{D}$ numerical methods.

In order to bring out the differences in $2 \mathrm{D}$ and $3 \mathrm{D}$ responses, the present work provides laboratory analogue model results for induction responses of elongated conductor models for a range of conductor lengths, overburden depths, and conductive basement depths, all for a wide period range. The results provided here are for a central profile normal to the strike of the conductor, where as in earlier related work, Chen and Dosso (1997) examined the responses of a fixed

Copy right (c) The Society of Geomagnetism and Earth, Planetary and Space Sciences (SGEPSS); The Seismological Society of Japan; The Volcanological Society of Japan; The Geodetic Society of Japan; The Japanese Society for Planetary Sciences. length conductor parallel to an ocean coastline, and Chen et al. (1997) studied the responses of a pair of elongated parallel conductors perpendicular to an ocean coastline for both central and near-end profiles over the conductor. In this earlier work, the prime purpose was to examine the validity of removing ocean coast effects by a simple vector subtraction of the coast effect induction arrows from the field survey induction arrows before carrying out an interpretation of the survey measurements.

The behavior of the in-phase induction arrows for 2D models has been well studied, with the arrow magnitudes known to be period dependent and at all periods to be pointing towards conductive bodies (or current concentrations). Further, the in-phase anomalous responses observed in the near vicinity of a conductive structure, show the maximum response occuring at a specific period that is characteristic of the model (Rokityansky, 1982). This period is known as the characteristic period $(T c)$ of the model. The behavior of the quadrature arrow, however, is not as well understood, particularily for 3D models. The quadrature arrow responses over the resistive host, but in the vicinity of the conductor-host interface, have been observed in various numerical and analogue model studies (e.g., Nienaber et al., 1983; Chen and Fung, 1987; Agarwal and Dosso, 1990, 1993, 2000; Chen and Dosso, 1997) to reach a minimum (or zero) at a characteristic period, and with the behavior that the arrows for periods $T<T c$ point away from the conductor-host interface, and for periods $T>T c$ are reversed in direction to point towards the interface. This quadrature response minimum (or zero) at the surface, accompanied by an in-phase response maximum, would require a specific induced current distribution in the conductive structure, in order that the resultant induced fields at the characteristic period be in phase with the induc- 
ing source field. Since the induced current distribution at a given period, both in the conductor and in the conductive structure of the host, is contolled by the skin depth effect in each component, clearly the embedded conductor dimensions, the overburden depth, and the conductive basement at depth beneath the conductor would be important parameters in determining the behavior of the induced anomalous fields observed at the surface in the vicinity of any embedded anomalous conductor. Thus, it is suggested that in practice the characteristic period, as well as the amplitudes of the induction arrow responses in the vicinity of an embedded conductor, could provide information on both the anomalous conductor and the conductive structure of the region.

In the present work, the responses of an elongated conductor of given length for a central profile can be expected to be those of 2D at sufficiently short periods, and to become progressively more 3D-like with increasing period. The response magnitudes as well as the characteristic periods $T c$ are expected to be different for 3D as compared with those for $2 \mathrm{D}$ structures. Further, the depths of the overburden and the conductive basement, as well as the conductor length, are expected to affect both the $2 \mathrm{D}$ and the $3 \mathrm{D}$ response characteristics. The array of laboratory analogue model results shown in the present work could provide suitable test values for 3D numerical methods for high conductivity contrast models (in the present case a conductor-host conductivity contrast of approximately 5800).

\section{The Laboratory Analogue Models}

Figure 1 shows the schematic cross-section of the elongated conductor $(3.6 \mathrm{~S} / \mathrm{m})$ embedded in a uniformly resistive host earth $\left(6.25 \times 10^{-4} \mathrm{~S} / \mathrm{m}\right)$ and underlain by a conductive

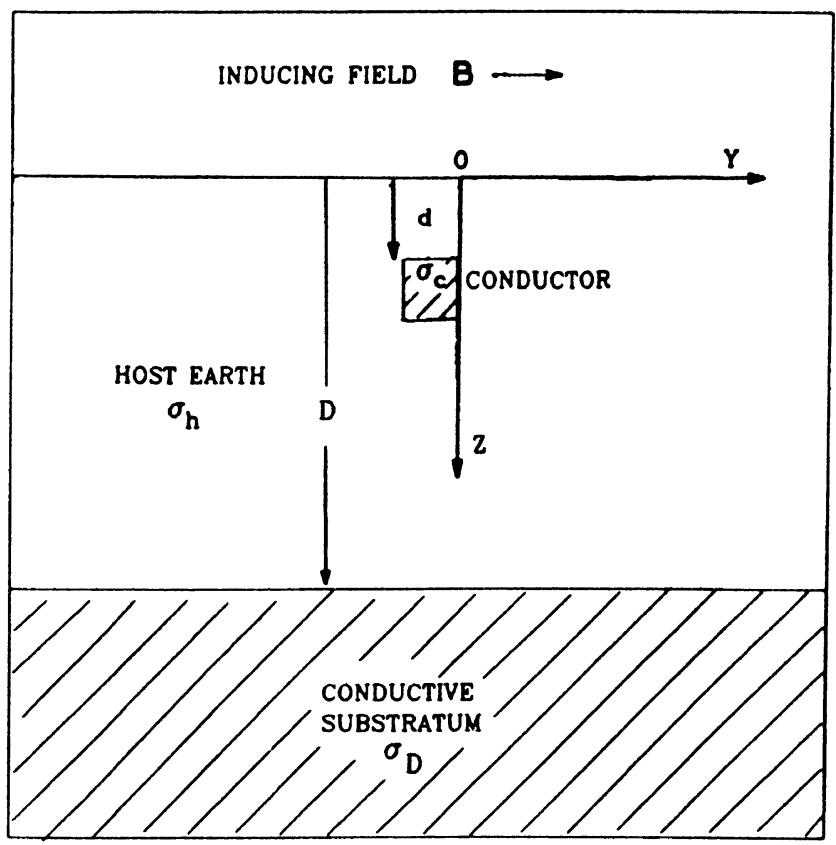

Fig. 1. Section view of the model of an elongated conductor (situated along the $x$-axis) of cross-section $50 \mathrm{~km} \times 50 \mathrm{~km}$ and length $L$, embedded at a depth $d$ in the host earth and underlain by a conductive substratum at depth $D$. The conductivities $\sigma_{c}, \sigma_{h}$, and $\sigma_{D}$ are $3.6,6.25 \times 10^{-4}$, and 3.6 $\mathrm{S} / \mathrm{m}$ respectively. substratum $(3.6 \mathrm{~S} / \mathrm{m})$ that was simulated in the laboratory. Graphite plate material was used to simulate the elongated conductor and the conductive substratum, while concentrated brine in a large wooden modelling tank simulated the host earth. The modelling facility, that includes the measuring equipment and the over head uniform field source, has been discussed previously (see for example, Dosso, 1973) and has since then been used in many analogue model studies at the University of Victoria. The frequency and length scaling factors were chosen so that $100 \mathrm{kHz}$ and $1 \mathrm{~mm}$ in the laboratory model would simulated a 5 min period and a $1 \mathrm{~km}$ length in the geophysical scale. In all models the cross-section of the elongated conductor was $50 \mathrm{~km} \times 50 \mathrm{~km}$, a rather major physical size chosen intentionaly so as to yield significant responses even for cases of large overburden depths.

For the elongated conductor models, the in-phase (real) and the quadrature (imaginary) magnetic field components of $B y$ and $B z$ were measured for a central profile $(x=0)$ in the $y$-direction (perpendicular to the strike of the conductor) at simulated periods of 2.5-90 min for the case of the over head uniform inducing $B$-field in the $y$-direction (Fig. 1). It could be noted that for a 2D model this would be known as the case of $E$-polarization inducing field. The $B y$ and $B z$ field components measured along the profile were used to calculate the well known Parkinson induction arrows, both

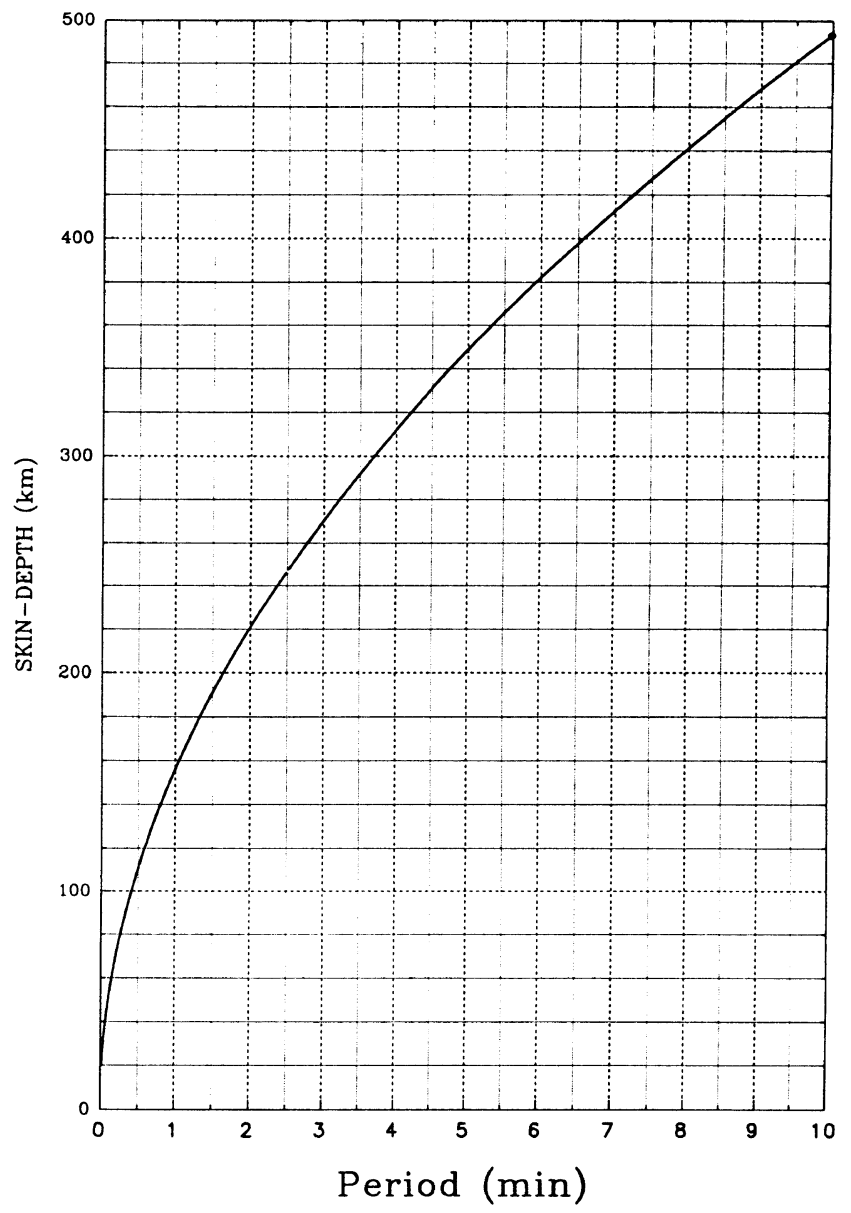

Fig. 2. The skin-depth $[\mathrm{km}]=[T / \pi \mu \omega]^{1 / 2} \times 10^{3} \mathrm{~km}$, where $T$ is in seconds, and $\sigma=6.25 \times 10^{-4} \mathrm{~S} / \mathrm{m}$ in the host medium. 
in-phase (real) and quadrature (imaginary), with the signs of both reversed following the convention of Lilley and Arora (1982) for time varying fields of the form $\exp [i \omega t]$. The $B x$ fields were not measured, since for a central profile the $B x$ component is everywhere zero. Model results are provided for elongated conductor lengths $L=500,1000,1500 \mathrm{~km}$; overburden depths $d=0,50,100,150,200 \mathrm{~km}$; and conductive basement depths $D=200,300,450,620 \mathrm{~km}$ for the period range $T=2.5-90 \mathrm{~min}$.

Since in the discussion of the results, frequent reference will be made to conductor lengths in terms of skin depths in the $6.25 \times 10^{-4} \mathrm{~S} / \mathrm{m}$ host medium, the Fig. 2 graph is included to readily provide these values for the $0-10 \mathrm{~min}$ period range of particular interest in the present work.

\section{Discussion of the Analogue Model Measure- ments}

Figure 3 shows the in-phase and the quadrature induction arrow responses $V y$, along the central profile for the model of conductor length $L=1500 \mathrm{~km}$, overburden depth $d=0$ $\mathrm{km}$, and conductive substratum depth $D=620 \mathrm{~km}$. In

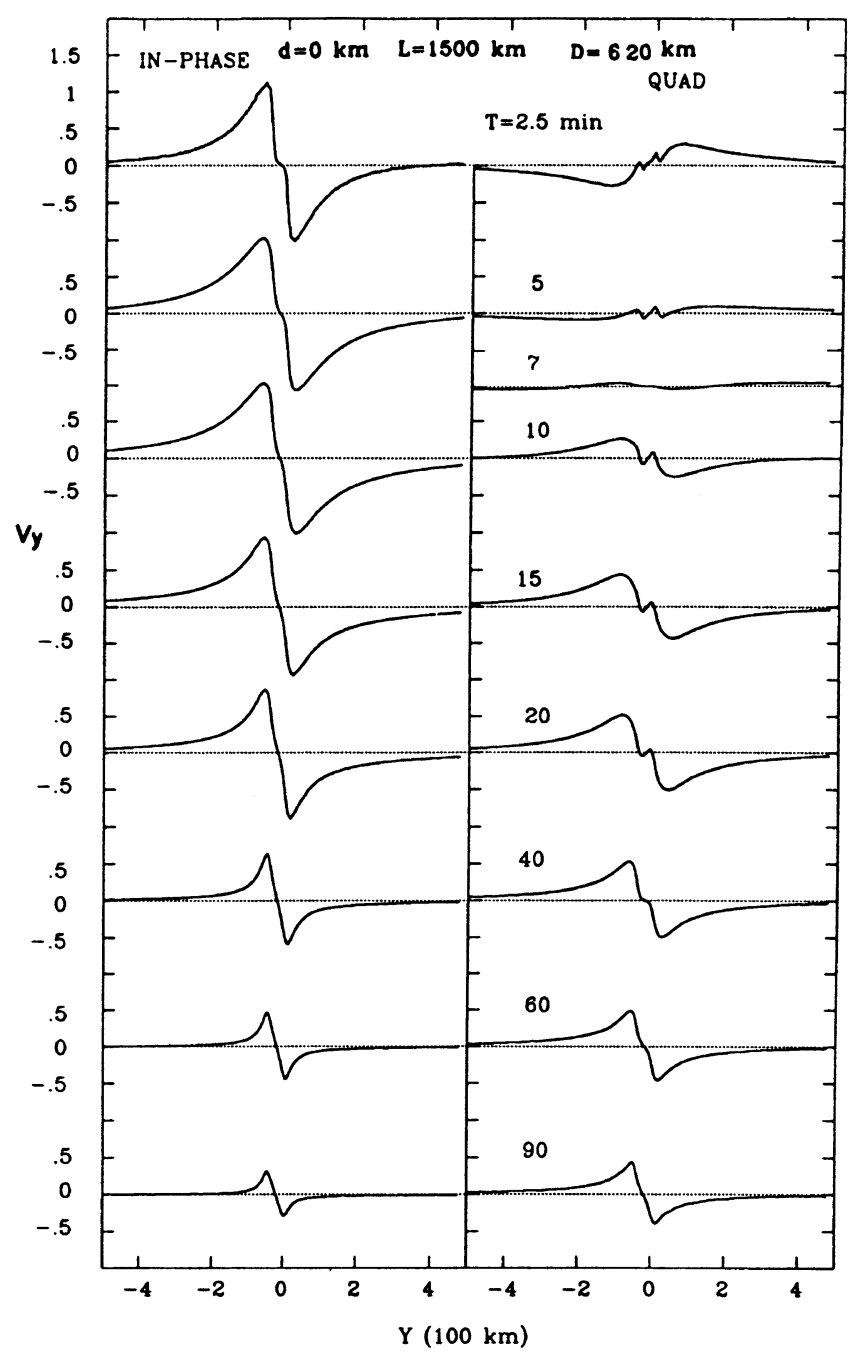

Fig. 3. The in-phase and quadrature $V y$ induction arrow responses for the central profile over the elongated conductor of length $L=1500 \mathrm{~km}$ for the case of no overburden $(d=0 \mathrm{~km})$. this figure positive values of the $V y$ response curve indicate arrows pointing in the positive $y$-direction, while negative values indicate arrows pointing in the negative $y$-direction. The two features of the in-phase response maximum and the quadrature response minimum (followed by quadrature sign reversal for $T>T c$ ), are clearly seen in Fig. 3, where to the right of the conductor $(y>0)$, the quadrature $V y$ response curve positive at $2.5 \mathrm{~min}$, has changed sign to be negative at $10 \mathrm{~min}$, the same period range where the in-phase anomalous response is maximum (with a value of roughly -1 ). The positive values of the response curve for $T<T c$ indicate quadrature arrows pointing away from the conductor-host interface, while the negative values for $T>T c$ indicate arrows pointing towards the conductor-host interface. In order to determine the value of Tc more closely, model measurements where carried out for 0.2 min period increments between the 5 and 10 min range. This leads to the characteristic period being approximately $7 \mathrm{~min}$ as shown by the $7 \mathrm{~min}$ quadrature response curve inserted between the 2.5 and 10 min curves in Fig. 3. In this curve, it is seen that the response is minimal over an extended spatial range along the profile, not only at the conductor-host boundary. The non-zero negative values (to the right of the conductor) even at $7 \mathrm{~min}$, would indicate that the actual characteristic period is somewhat less than $7 \mathrm{~min}$.

At short periods in the neighborhood of $7 \mathrm{~min}$, the 1500 $\mathrm{km}$ length conductor would be expected to show approximate $2 \mathrm{D}$ response characteristics, and thus the relatively constant in-phase response value (approximately -1 ) in the period range $2.5-10 \mathrm{~min}$ shown in Fig. 3 would be expected. To be a truly $2 \mathrm{D}$ response, the conductor length should be of infinite length. However, if for practical purposes the conductor length criteria for a $2 \mathrm{D}$ response is taken to be at least 4 skin depths in terms of the host conductivity, the 1500 $\mathrm{km}$ long conductor would be expected to show at least approximate 2D-like responses at periods $T<7 \mathrm{~min}$, since the conductor length at $7 \mathrm{~min}$ is roughly 4 skin depths in the $6.25 \times 10^{-4} \mathrm{~S} / \mathrm{m}$ host medium. With increasing period beyond the $7 \mathrm{~min}$ characteristic period, the responses are expected to be increasingly more $3 \mathrm{D}$ than $2 \mathrm{D}$. This is supported by the essentially identical in-phase responses at the 2.5-10 min range in Fig. 3, and the fairly rapidly decreasing responses with increasing period beyond $10 \mathrm{~min}$. At 90 min the in-phase responses are seen to be greatly reduced as would be expected, since the $1500 \mathrm{~km}$ length at this period has been reduced to about 1 skin depth (see Fig. 2), and thus the responses are certainly no longer that of a 2D structure. It is also interesting to note that the quadrature $V y$ responses in Fig. 3 at periods greater than $7 \mathrm{~min}$, are seen to increase to attain maximum response between 15 and $20 \mathrm{~min}$, and then to decrease more gradually than the in-phase responses, so that at $90 \mathrm{~min}$ the quadrature response is significantly larger than the in-phase response.

The detailed measurements (not given here) also carried out for conductor lengths $L=1000 \mathrm{~km}$ and $500 \mathrm{~km}$, showed response curves similar in shape to those of Fig. 3, but with both the characteristic periods and the in-phase responses decreased for these shorter lengths $L$, as the conductor responses become more 3D-like. The effect of decreasing the conductive basement depth $(D=450,300,200 \mathrm{~km})$ is also 
seen to decrease both the characteristic periods and the magnitudes of the in-phase responses. The array of characteristic periods and the in-phase response maxima obtained from the detailed model measurements are given in empirical curves in figures that follow.

To provide an example of the effect of adding an overburden, Fig. 4 shows the in-phase and the quadrature $V y$ responses for the model of conductor length $L=1500 \mathrm{~km}$ and conductive basement depth $D=620 \mathrm{~km}$, for the case of overburden depth $d=50 \mathrm{~km}$. Due to the increased depth below the surface at which the currents are induced in the elongated conductor, the responses are much attenuated and smoothed, as expected, compared with the responses for zero overburden $(d=0 \mathrm{~km})$ as shown in Fig. 3. In the detailed measurements carried out in increments of $0.2 \mathrm{~min}$ (not shown here), the $T c$ period was determined to be approximately $8.5 \mathrm{~min}$ as compared with $7 \mathrm{~min}$ in Fig. 3 for the case of $d=0 \mathrm{~km}$. The effect of the overburden is thus seen to somewhat increase the $T c$ value, also due to the increased depth below the surface at which the currents are induced in the conductor for the case of an overburden. The detailed

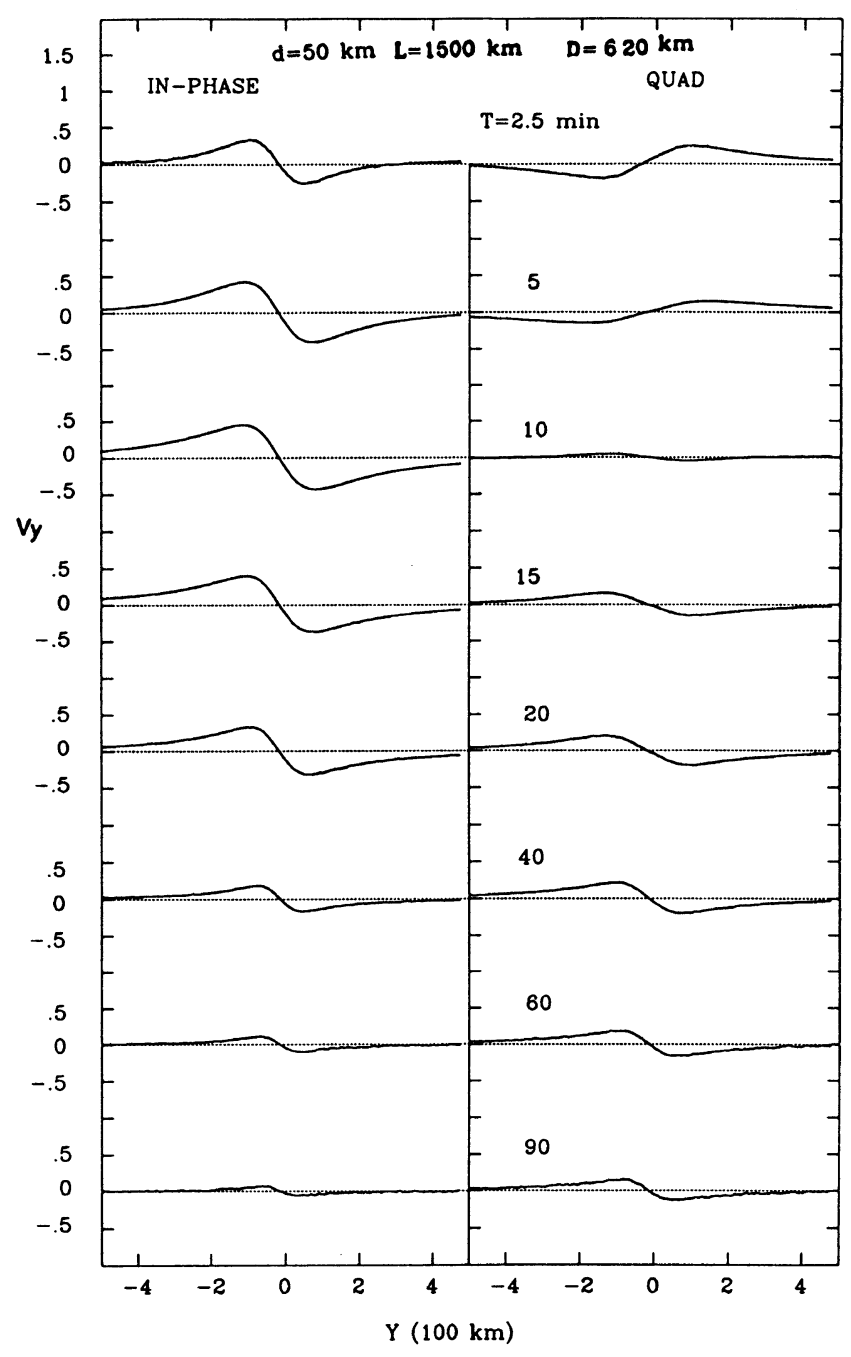

Fig. 4. The in-phase and quadrature $V y$ induction arrow responses for the central profile over the elongated conductor of length $L=1500 \mathrm{~km}$ for the case of overburden depth $d=50 \mathrm{~km}$.
$T c$ dependence on conductor length and overburden depth is included in empirical curves that follow.

Figure 5, showing empirical curves of the characteristic period $T c$ as a function of overburden depth $d$, provides a simple summary of the effects of conductor length $L$ and conductive basement depth $D$. The general observations in the Fig. 5 results are that decreasing the conductor length or the conductive basement depth, each have the effect of decreasing the characteristic period $T c$, while increasing the over burden depth $d$ increases the characteristic period $T c$. Clearly, the characteristic period for any given over burden depth does not depend on the conductive basement depth alone, or the conductor length alone, but on the net effect of the distribution of induced currents in the various components of the conductive structure. As examples of this, in the absence of any over burden $(d=0 \mathrm{~km})$ and with a fixed basement depth $D=620 \mathrm{~km}$, the characteristic period decreases from approximately $7 \mathrm{~min}$ for $L=1500 \mathrm{~km}$, to $6 \mathrm{~min}$ and 4 min for $L=1000 \mathrm{~km}$ and $500 \mathrm{~km}$ respectively, while for a fixed length $L=1500 \mathrm{~km}$ the characteristic period changes from approximately $7 \mathrm{~min}$ for the basement depth $D=620$ $\mathrm{km}$, to 6,5 , and $3.5 \mathrm{~min}$ for decreased depths $D=450,300$, $200 \mathrm{~km}$ respectively. Further, even for a geophysically more realistic basement depth $D=200 \mathrm{~km}$, and in the absence of overburden $(d=0)$, it is apparent that the earlier proposed conductor length criteria of 4 skin depths does not ensure a fully 2D-like response, since the characteristic periods which

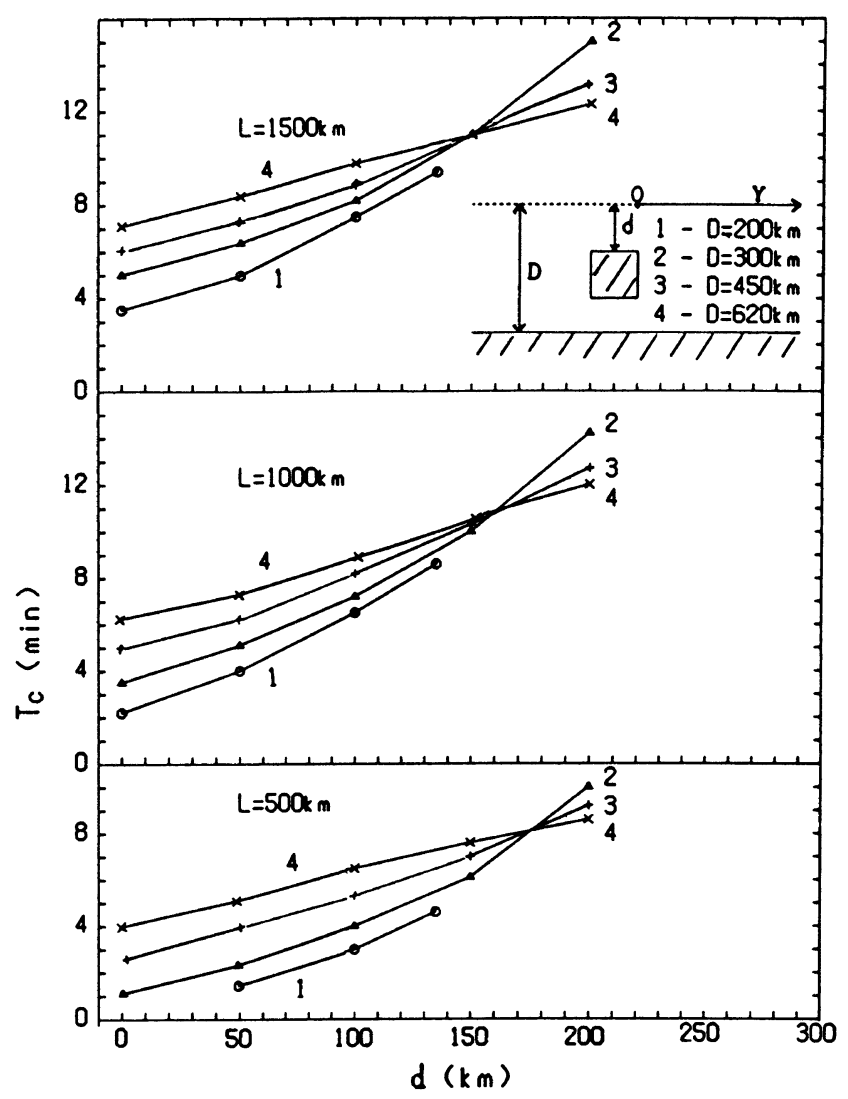

Fig. 5. Empirical curves of the characteristic period $T c$ as a function of overburden depth $d$ for conductor lengths $L=1500,1000,500 \mathrm{~km}$, and conductive substratum depths $D=200,300,450$, and $620 \mathrm{~km}$. 
should not change for perfect 2D responses, do in fact change noticeably. It is seen that the periods are roughly $3.5,2.2$, and somewhat less than $0.5 \mathrm{~min}$, with corresponding skin depths of approximately 290, 225, and $120 \mathrm{~km}$ (see skin depths in Fig. 2) for the conductor lenghts $L=1500,1000$, and 500 $\mathrm{km}$, respectively. At their respective characteristic periods, the 1500,1000 , and $500 \mathrm{~km}$ length conductors in terms of skin depths are roughly 5.2, 4.4, and 4.2 respectively, with each thus at least of 4 skin depths in length. In earlier work, Chen and Dosso (1997) found that for a simulated ocean 1000 $\mathrm{km}$ long, $500 \mathrm{~km}$ wide, and $5 \mathrm{~km}$ deep, the characteristic period was $2.6 \mathrm{~min}$ for a model with basement depth $D=200$ $\mathrm{km}$, as compared with approximately $2.2 \mathrm{~min}$ (see curve 1 for $d=0 \mathrm{~km}$ and $L=1000 \mathrm{~km}$ in Fig. 5) for the present conductor of the same length $L=1000 \mathrm{~km}$, but of cross-section $50 \mathrm{~km} \times 50 \mathrm{~km}$. This difference in characteristic periods could be accounted for by the differing geometry of the two models, with the present elongated conductor, though of the same $1000 \mathrm{~km}$ length, being a factor of 10 narrower and a factor of 10 thicker than the ocean model of Chen and Dosso (1997).

Another point of note in Fig. 5 is the cross-over of the curves seen for each value of $L$, for example, the crossover at roughly $d=150 \mathrm{~km}$ and $T c=11 \mathrm{~min}$ for the $L=1500 \mathrm{~km}$ model. This behavior suggests that for a given conductor length, the $T c$ value at the cross-over point should, suprisingly, not depend on the basement depth $D$.

Figure 6, providing the companion set of results for Fig. 5, shows empirical curves of the response maximum $(V y c)$ at the characteristic period $(T c)$ as a function of overburden depth $d$. It also provides a summary of the dependence on

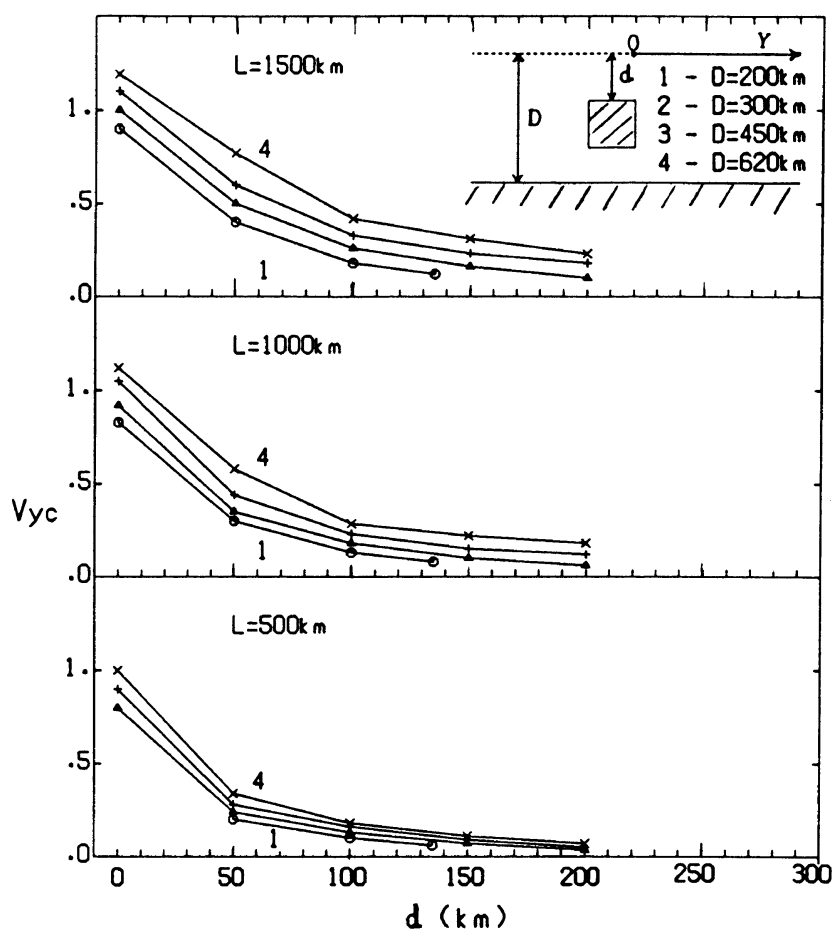

Fig. 6. Empirical curves of the in-phase response maximum $V y c$ at the characteristic period as a function of overburden depth $d$ for conductor lengths $L=1500,1000,500 \mathrm{~km}$ and conductive substratum depths $D=1500,1000,500 \mathrm{~km}$. conductor length $L$ and conductive basement depth $D$. The general observations are, that the effect of decreasing the conductor length $L$ or the conductive basement depth $D$, or increasing the overburden depth $d$, is in each case to decrease the in-phase response $V y c$.

A somewhat different suite of empirical curves are provided in Fig. 7, which are obtained by simply plotting the maximum in the anomaly of each of the in-phase and the quadrature responses observed in the vicinity of the conductor-host interface at the periods $2.5,5,10,20,40,60$, and $90 \mathrm{~min}$. The intersection of the quadrature $V y$ response curves and the zero response axis, where the response reverses sign, defines the characteristic period for each model. Curves of this form would, in fact, yield the $T c$ values and the in-phase response maxima more simply and more consistently than obtained by carrying out numerous measurements for the fine period detail of 0.2 period increments as described for the Fig. 3 and Fig. 5 results. In Fig. 7, it can be seen that
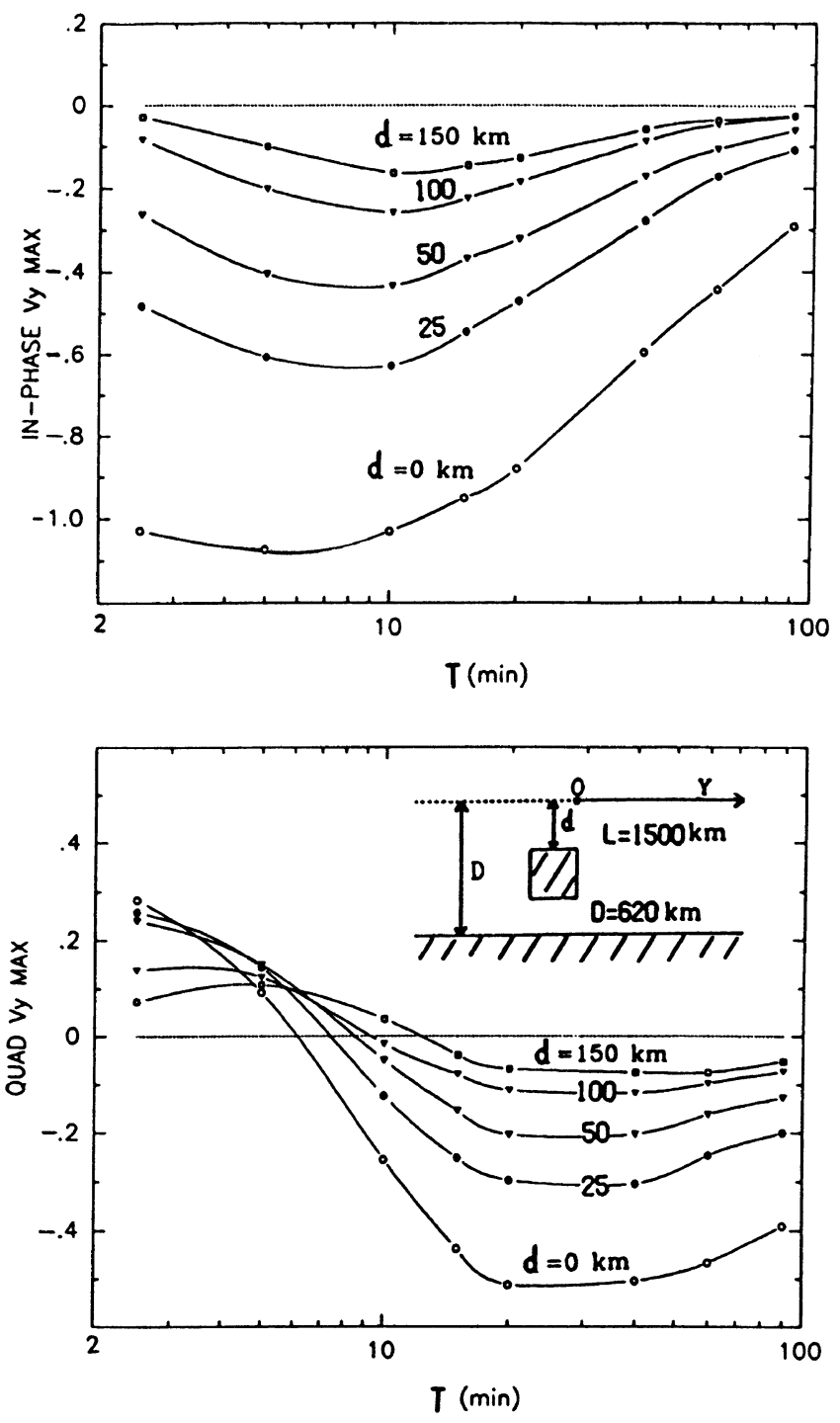

Fig. 7. The in-phase and quadrature maximum $V y$ responses as a function of period for a range of overburden depths $d=0,25,50,100,150 \mathrm{~km}$ for the elongated conductor of length $L=1500 \mathrm{~km}$ and substratum depth $D=620 \mathrm{~km}$ 
the $T c$ periods obtained in this way are roughly the same as those determined by the detailed period measurements used in the previous figures. (It should be realized that more points on these curves would be needed in order to provide more accurate empirical curves than the extrapolated smooth hand-drawn curves shown here.) The differences in the Fig. 5 and Fig. $7 T c$ values could in part be accounted for as due to the difficulty in determining the precise period at which the quadrature response is minimum on the basis of detailed period model measurements, and the accuracy at which these empirical curves can be drawn using only a limited number of measurements. It is also noted that as expected, the maximum in the in-phase empirical curves in Fig. 7, which should be the $V y c$ values, occur at roughly the same periods $(T c)$ as the quadrature reversals. Thus, empirical curves of the form shown in Fig. 7, can readily provide the approximate characteristic periods $T c$, as well as the maximum in-phase anomalous responses at the characteristic periods, from an empirical plot of the measured anomalous responses at an arbitrary set of periods in the neighborhood of the characteristic period.

\section{Conclusions}

For models of an elongated conductor embedded in a resistive host and underlain by a conductive basement, the characteristic period $T c$, the period at which the in-phase anomalous response is maximum and the quadrature response is minimum and reverses sign, were found to depend on the conductor length, the overburden depth, and the conductive basement depth. Specifically, decreasing the conductor length or the conductive basement depth had the effect of decreasing both the response amplitude and the characteristic period, where as increasing the overburden depth lead to decreasing the response amplitude and increasing the characteristic period. Further, while the elongated conductor showed a 2D response at sufficiently short periods where the elongated conductor was at least 4 skin depths long in the host conductivity, the rapid response fall-off with increasing period greater than $T c$, demonstrated the expected $3 \mathrm{D}$, rather than a $2 \mathrm{D}$ response, at the longer periods. It can readily be seen that a determination of the characteristic period and the accompanying in-phase anomalous response maximum, could in practice yield information on the embedded conductor and the conductivity structure of a survey region. Since a number of physical parameters (in addition to conductivities) are required to define any conductive structure of a region, some of the parameters would need to be constrained to permit an interpretation. For example, if the conductor length and the overburden depth for a region (described by the simple model under study here) were known, then obtaining the characteristic period would provide information on the conductive basement depth, or alternatively, if the depths of the overburden and the conductive basement were known, the characteristic period could provide information on the conductor length.

It would be of interest to examine further the effect of the conductor dimensions (for example, the length to width, and the length to depth ratios), as well as the effect of different conductor-host conductivity contrasts, with the aid of a proven 3D numerical modelling method. The laboratory analogue modelling facility at the University of Victoria would have permitted further study of some aspects of this problem, but the laboratory was disbanded after the retirement of $\mathrm{H}$. W. Dosso in 1999.

Acknowledgments. We thank Laszlo Szarka and Toru Mogi for their reviews and constructive comments. The financial support of an NSERC grant (H.W.D.) is gratefully acknowledged.

\section{References}

Agarwal, A. K. and H. W. Dosso, On the behaviour of the induction arrows over a buried conductive plate-a numerical model study, Phys. Earth Planet. Inter., 60, 265-277, 1990.

Agarwal, A. K. and H. W. Dosso, The characteristic periods of the induction arrows for a conductive-resistive vertical interface-a numerical model study, Phys. Earth Planet. Inter., 7, 67-74, 1993.

Agarwal, A. K. and H. W. Dosso, EM induction in a large conductive blocka 3D numerical study, 2000 (submitted).

Chen, J. and H. W. Dosso, EM responses of an elongated conductor near an ocean-analogue model studies, Phys. Earth Planet. Inter., 99, 83-89, 1997.

Chen, J., H. W. Dosso, and S. Kang, EM induction in elongated conductors normal to a coastline with application to geomagnetic measurements in Nigeria, J. Geomag. Geoelectr., 49, 1401-1414, 1997.

Chen, P. F. and P. C. W. Fung, On the behaviour of the imaginary Parkinson arrows near the anomalous conductive-host medium interface, Phys. Earth Planet. Inter., 50, 195-198, 1987.

Dosso, H. W., A review of analogue model studies of the coast effect, Phys. Earth Planet. Inter., 7, 294-302, 1973.

Lilley, F. E. M. and B. R. Arora, The sign convention for Parkinson arrows in geomagnetic induction studies, Rev. Geophys. Space Phys., 20, 513-518, 1982.

Nienaber, W., D. Hebert, and H. W. Dosso, Induction arrows for a buried conducting plate, Phys. Earth Planet. Inter., 32, 306-311, 1983.

Rokityansky, I. I., Geoelectromagnetic Investigation of the Earth's Crust and Mantle, Springer-Verlag, Berlin, 287 pp., 1982.

H. W. Dosso (e-mail: hdosso@uvic.ca) and J. Chen 